\title{
Synthesis and evaluation of antibacterial activity for a series of $N$-phthaloylglycine derivatives
}

\author{
Samreen Begum a, Shaikh Sirajuddin Nizami a, Sumayya Saied a, \\ Syed Muhammad Shahid ${ }^{\mathrm{b}}$ and Fatima Zehra Basha ${ }^{\mathrm{c}, *}$ \\ a Department of Chemistry, University of Karachi, Karachi-75270, Pakistan \\ b The Karachi Institute of Biotechnology and Genetic Engineering (KIBGE), University of Karachi, Karachi-75270, Pakistan \\ c Hussain Ebrahim Jamal Research Institute of Chemistry, International Center for Chemical and Biological Sciences, University of Karachi, Karachi-75270, Pakistan
}

${ }^{*}$ Corresponding author at: Hussain Ebrahim Jamal Research Institute of Chemistry, International Center for Chemical and Biological Sciences, University of Karachi, Karachi-75270, Pakistan.

Tel.: +92.21.99261767. Fax: +92.21.4819018. E-mail address: bashafz@gmail.com (F.Z. Basha).

\section{ARTICLE INFORMATION}

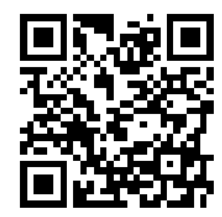

DOI: $10.5155 /$ eurjchem.5.4.557-562.1077

Received: 19 April 2014

Received in revised form: 16 June 2014

Accepted: 16 June 2014

Online: 31 December 2014

\section{KEYWORDS}

\section{Antibacterial}

Benzimidazole

Phthaloylglycine derivatives

Schotten-Baumann reaction

Methicillin-resistant Staphylococcus aureus

Vancomycin-resistant Staphylococcus aureus

\section{Introduction}

The frequent use and misuse of most antibiotics, in particular, methicillin and vancomycin, has caused many bacteria to develop resistance to these agents $[1,2]$. Antibacterial resistance has increased globally, and has emerged as a serious medical challenge worldwide. Therefore, the development of novel antibacterial agents to treat patients infected with methicillin-resistant Staphylococcus aureus (MRSA) and vancomycin-resistant Staphylococcus aureus (VRSA), in addition to many other drug resistant microbes remains an important challenge [3-7].

$\mathrm{N}$-Phthaloyl derivatives of amino acids and benzimidazoles are important pharmacophores of a number of medicinally important classes of compounds. Benzimidazole derivatives are very useful intermediates of pharmaceutically important compounds [8]. They are applicable as antiulcer, antihypertensive, antifungal, antiviral, antihistamine, antimicrobial, antiallergic, antioxidant, antitubercular, and in-vitro anti-HIV-1 [9]. Likewise, derivatives of $N$-phthaloyl amino acids have demonstrated a number of biological activities, including antimicrobial [10], hypolipidemic [11], analgesic [12] and DNA cleaving abilities [13]. In addition, their amides represent a new class of antiepileptic agents [14]. The derivatives of $\mathrm{N}$-phthaloylglycine have been studied most widely, including the formation of complexes with different transition metals [15] and adducts formation with different aromatic amines [16]. Some heterocyclic derivatives of $N$-phthaloyl amino acids, such as oxadiazole, benzoxinone, 1,2,4-triazoles are also reported $[17,18]$.

In this context, we were intrigued by the number of reports that derivatives of $N$-phthaloylglycine and benzimidazole have a wide range of important biological activities. Consequently, we synthesized analogues that contain both pharmacophores in the framework of one molecule. In addition, the synthesized $\mathrm{N}$-phthaloylglycine derivatives were subjected to antibacterial activity. Herein we report the result of that study and discuss their structure-activity relationship.

\section{Experimental}

\subsection{Instrumentation}

The melting points of all the compounds were obtained with digital Electro thermal equipment and are uncorrected. 


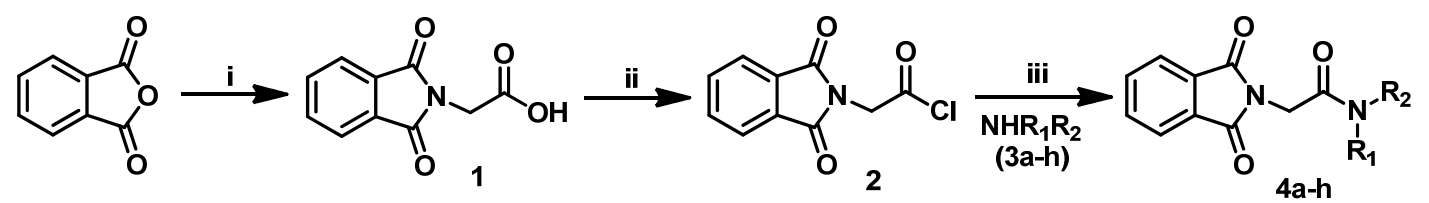

\author{
Reagents and conditions: \\ i) Glycine, heat \\ ii) $\mathrm{SOCl}_{2}$, reflux \\ iii) Sodium acetate, $\mathrm{CHCl}_{3}$, rt
}

\begin{tabular}{lll} 
Compounds & $\mathrm{R}_{1}$ & $\mathrm{R}_{2}$ \\
\hline $3 \mathrm{a} / 4 \mathrm{a}$ & $\mathrm{CH}_{3}$ & $\mathrm{CH}_{3}$ \\
$3 \mathrm{~b} / 4 \mathrm{~b}$ & $\mathrm{C}_{2} \mathrm{H}_{5}$ & $\mathrm{C}_{2} \mathrm{H}_{5}$ \\
$3 \mathrm{c} / 4 \mathrm{c}$ & $\mathrm{i}$-But & $i-\mathrm{But}$ \\
$3 \mathrm{~d} / 4 \mathrm{~d}$ & $\mathrm{H}$ & $\mathrm{C}_{3} \mathrm{H}_{7}$ \\
$3 \mathrm{e} / 4 \mathrm{e}$ & $\mathrm{H}$ & $\mathrm{C}_{5} \mathrm{H}_{11}$ \\
$3 \mathrm{f} / 4 \mathrm{f}$ & $\mathrm{H}$ & $p-$ Methoxybenzene \\
$3 \mathrm{~g} / 4 \mathrm{~g}$ & $\mathrm{H}$ & Pyridine \\
$3 \mathrm{~h} / 4 \mathrm{~h}$ & $\mathrm{H}$ & Ethyl alcohol
\end{tabular}

Scheme 1
${ }^{1} \mathrm{H}$ NMR Spectra were recorded on Avance Bruker 300, 400 $\mathrm{MHz}$ in $\mathrm{CDCl}_{3} .{ }^{13} \mathrm{C}$ NMR were recorded on Bruker Aspect $\mathrm{AB}-$ 100 MHz. The EI-MS were measured on Finnigan MAT-312, Germany, and JEOL MS Route JMS. 600H, Japan Instruments. TLC Analysis was performed on pre-coated silica gel aluminum plates (Kieselgel $60 \mathrm{~F}_{254}$, E. Merck, Germany).

\subsection{Synthesis}

The synthesis of $N$-phthaloylglycine amides (4a-h) was carried out from commercially available phthalic anhydride (Scheme 1). Heating an equimolar mixture of phthalic anhydride and glycine afforded the $\mathrm{N}$-phthaloylglycine $\mathbf{1}$ in $72 \%$ yield [19]. Subsequently, compound $\mathbf{1}$ was converted into corresponding acid chloride 2 by treating with thionyl chloride $\left(\mathrm{SOCl}_{2}\right)$. The resulting phthaloylglycyl chloride (2-(1,3-dioxo isoindolin-2-yl)acetyl chloride) $\mathbf{2}$ is treated with different amines (3a-h) to give the $N$-phthaloylglycine amides $(\mathbf{4 a - h})$ in $30-32 \%$ yield (Scheme 1). The substituted benzimidazole derivatives (5a-d) were synthesized by treating $o$-phenylene diamine with different carboxylic acids (Scheme 2) [19]. To synthesize the desired benzimidazole derivatives of $N$-phtha loylglycine (6a-d), we utilized the environmentally benign Schotten-Baumann reaction condition (Scheme 3) [20].

\subsubsection{Procedure for the preparation of $N$-phthaloylglycine (1)}

A well pulverized mixture of phthalic anhydride $(6.0 \mathrm{~g}, 0.02$ moles) and glycine (3.0 g, 0.02 moles) was heated with a burner until the solid melted. The molten mass was stirred gently with a glass rod, and heated again at $150-190{ }^{\circ} \mathrm{C}$ for 15 minutes. The mixture was allowed to cool and then recrystallized with water $(100 \mathrm{~mL})$ (Scheme 1) [21].

N-Phthaloylglycine (1) [21]: Color: White. Yield: 72\%. M.p.: 196-198 ${ }^{\circ} \mathrm{C} . \mathrm{R}_{f}: 0.78$ (EtOAc:MeOH) (9:1). FT-IR (KBr, $\left.v, \mathrm{~cm}^{-1}\right)$ : 3450-3000 (OH) (br, carboxylic acid), 1770, $1721 \quad(\mathrm{C}=0)$ (imide), 1669 ( $\mathrm{C}=0$ ) (acid), 1560 (Ar C=C), 1398 (C-0), 711 (ortho substituted Ar). ${ }^{1} \mathrm{H}$ NMR (300 MHz, DMSO- $d_{6}, \delta, \mathrm{ppm}$ ): $4.30\left(\mathrm{~s}, 2 \mathrm{H}, \mathrm{N}-\mathrm{CH}_{2}-\mathrm{C}=0\right), 7.85-7.93(\mathrm{~m}, 4 \mathrm{H}, \mathrm{Ar}-\mathrm{H}), 13.22(\mathrm{~s}, 1 \mathrm{H}$, OH). MS (EI, $m / z(\%)): 205\left(\mathrm{M}^{+}, 10\right), 160(100), 149(4), 133.0$ (70), 104 (70), 77 (65), 50 (30).

\subsubsection{Procedure for the preparation of $N$-phthaloylglycyl chloride (2)}

Pure phthaloylglycine (1) (2.4 g) was refluxed with $\mathrm{SOCl}_{2}$ $(9.0 \mathrm{~mL})$ for $1 \mathrm{~h}$. The condenser was removed and the excess $\mathrm{SOCl}_{2}$ was evaporated from the reaction mixture (Scheme 1) [21].
N-Phthaloylglycyl chloride (2-(1,3-dioxoisoindolin-2-yl)acetyl chloride) (2) [21]: Color: Light yellow. Yield: 96\%. M.p.: 82-84 ${ }^{\circ} \mathrm{C} . \mathrm{R}_{f}$ : 0.75 (EtOAc:MeOH) (9:1). FT-IR (KBr, v, $\mathrm{cm}^{-1}$ ): 1755, $1765(\mathrm{C}=0)$ (imide), $1750(\mathrm{C}=0)$ (acid chloride), $1654(\mathrm{Ar} \mathrm{C}=\mathrm{C})$, 711 (ortho subst. Ar), 605 (C-Cl). ${ }^{1} \mathrm{H}$ NMR $\left(300 \mathrm{MHz}, \mathrm{DMSO}-d_{6}\right.$, $\delta, \mathrm{ppm}): 4.30\left(\mathrm{~s}, 2 \mathrm{H}, \mathrm{N}-\mathrm{CH}_{2}-\mathrm{CO}\right), 7.85-7.93(\mathrm{~m}, 4 \mathrm{H}, \mathrm{Ar}-\mathrm{H})$.

\subsubsection{Procedure for the preparation of benzimidazole (5a) and its derivatives ( $5 b-d)$}

The $o$-phenylenediamine $(10.0 \mathrm{~g}, 0.09 \mathrm{moles})$ was treated with appropriate carboxylic acid ( 0.135 moles). The mixture was heated at $100{ }^{\circ} \mathrm{C}$ for $2 \mathrm{~h}$. After cooling, $10 \% \mathrm{NaOH}$ solution was added slowly until the mixture was just alkaline to litmus. The crude product was collected, washed with cold water and recrystallized without drying. Recrystallization was done by dissolving in boiling water then treated with a $2 \mathrm{~g}$ of charcoal for 10 to 15 minutes and filtered the hot mixture which gave pure crystals of Benzimidazole on cooling (Scheme 2) [19].

1H-Benzo[d]imidazole (5a) [19,22]: Color: White. Yield: 50\%. M.p.: 170-172 ${ }^{\circ} \mathrm{C} . \mathrm{R}_{f}: 0.75$ (EtOAc:MeOH) (8:2). FT-IR (KBr, v, cm-1): $3414(\mathrm{~N}-\mathrm{H}$ stretching), $2620(\mathrm{C}-\mathrm{H})$ (aromatic), $1618\left(\mathrm{C}=\mathrm{N}\right.$, stretch), $1610(\mathrm{C}=\mathrm{C})$ (aromatic). ${ }^{1} \mathrm{H}$ NMR $(300 \mathrm{MHz}$, MEOD, $\delta, \mathrm{ppm}): 4.85(\mathrm{~s}, 1 \mathrm{H}, \mathrm{N}-\mathrm{H}), 7.26-7.21(\mathrm{q}, J=3.6 \mathrm{~Hz}, 2 \mathrm{H}$, Ar-H), 7.60-7.57 (q, $J=3.3 \mathrm{~Hz}, 2 \mathrm{H}, \mathrm{Ar}-\mathrm{H}), 8.12(\mathrm{~s}, 1 \mathrm{H}, \mathrm{N}=\mathrm{CH}-\mathrm{N})$. MS (EI, $m / z(\%)$ ): $118\left(\mathrm{M}^{+}, 100\right), 91$ (75), 63 (75), 52 (45).

2-Methyl-1H-benzo[d]imidazole (5b) [22,23]: Color: Light yellow. Yield: $35 \%$. M.p.: $174-176{ }^{\circ} \mathrm{C}$. Rf: 0.78 (EtOAc:MeOH) (8:2). FT-IR (KBr, v, $\left.\mathrm{cm}^{-1}\right)$ : $3412(\mathrm{~N}-\mathrm{H}$ stretching), $2677(\mathrm{C}-\mathrm{H})$ (aromatic), $1620\left(\mathrm{C}=\mathrm{N}\right.$, stretch), $1448(\mathrm{C}=\mathrm{C})$ (aromatic). ${ }^{1} \mathrm{H}$ NMR (300 MHz, MeOD, $\delta$, ppm): $2.53\left(\mathrm{~s}, 3 \mathrm{H}, \mathrm{CH}_{3}\right), 4.85$ (s, $1 \mathrm{H}, \mathrm{N}-$ $\mathrm{H})$, 7.16-7.13 (q, $J=3.3 \mathrm{~Hz}, 2 \mathrm{H}, \mathrm{Ar}-\mathrm{H}), 7.46-7.43(\mathrm{q}, J=3.3 \mathrm{~Hz}$ 2H, Ar-H). MS (EI, $m / z(\%)): 132\left(\mathrm{M}^{+}, 100\right), 104$ (12), 90 (15), 77 (10), 63 (30), 51.9 (18).

2-Ethyl-1H-benzo[d]imidazole (5c) [22]: Color: White. Yield: 32\%. M.p.: $178-179{ }^{\circ} \mathrm{C}$. Rf: 0.65 (EtOAc:MeOH) (8:2). FT-IR $\left(\mathrm{KBr}, \mathrm{v}, \mathrm{cm}^{-1}\right): 3412(\mathrm{~N}-\mathrm{H}$ stretching), $2700(\mathrm{C}-\mathrm{H})$ (aromatic), 1620 (C=N, stretch), 1610 (C=C) (aromatic). ${ }^{1} \mathrm{H}$ NMR $(300 \mathrm{MHz}$, MeOD, $\delta, \mathrm{ppm}): 1.41-1.36\left(\mathrm{t}, J=7.5 \mathrm{~Hz}, 3 \mathrm{H}, \mathrm{CH}_{3}\right), 2.93-2.86(\mathrm{q}, J$ $\left.=7.5 \mathrm{~Hz}, 2 \mathrm{H}, \mathrm{CH}_{2}\right), 4.83(\mathrm{~s}, 1 \mathrm{H}, \mathrm{N}-\mathrm{H}), 7.19-7.14(\mathrm{q}, J=3.6 \mathrm{~Hz}, 2 \mathrm{H}$ Ar-H), 7.48-7.45 (q, J = 3.0 Hz, 2H, Ar-H). MS (EI, $m / z$ (\%)): 145 ( $\left.\mathrm{M}^{+}, 100\right), 131$ (25), 118 (22), 77 (52), 65 (56), 63.1 (90), 52.1 (70).

1-(1H-Benzo[d]imidazole-2yl)ethanol (5d) [24,25]: Color: Yellow. Yield: $50 \%$. M.p.: $178-180{ }^{\circ} \mathrm{C}$. Rf: $0.78\left(\mathrm{CHCl}_{3}: \mathrm{EtOAc}\right)$ (8:2). FT-IR (KBr, $\left.v, \mathrm{~cm}^{-1}\right)$ : $3411(\mathrm{~N}-\mathrm{H}$, stretching), 3300-2562 (br, OH) (alcohol), 2352 (C-H) (aromatic), 1621 (C=N, stretch), $1591(\mathrm{C}=\mathrm{C})$ (aromatic). ${ }^{1} \mathrm{H}$ NMR $(300 \mathrm{MHz}, \mathrm{MeOD}, \delta, \mathrm{ppm})$ : 1.61-1.59 (d, $\left.J=6.6 \mathrm{~Hz}, 3 \mathrm{H}, \mathrm{CH}_{3}\right), 3.30(\mathrm{~s}, 1 \mathrm{H}, \mathrm{OH}), 4.83(\mathrm{~s}, 1 \mathrm{H}$ $\mathrm{N}-\mathrm{H}), 5.08-5.02(\mathrm{q}, J=6.6 \mathrm{~Hz}, 1 \mathrm{H}, \mathrm{CH}), 7.19-7.16(\mathrm{q}, J=3.3 \mathrm{~Hz}$, 


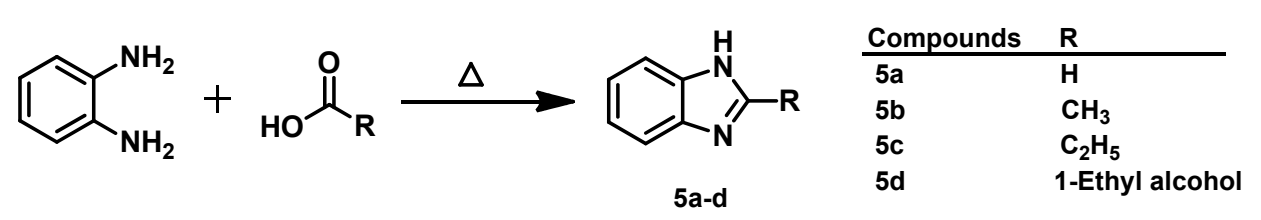

Scheme 2

$2 \mathrm{H}, \mathrm{Ar}-\mathrm{H}), 7.53-7.50$ (q, $J=3.0 \mathrm{~Hz}, 2 \mathrm{H}, \mathrm{Ar}-\mathrm{H}) . \mathrm{MS}(\mathrm{EI}, m / z(\%))$ : $160\left(\mathrm{M}^{+}, 50\right), 145$ (15), 132 (25), 118 (55), 82.9 (100), 43 (78).

\subsubsection{General procedure for the preparation of $N$-phthaloyl glycine amides (4a-h)}

To a solution of amines $\mathbf{3 a}-\mathbf{h}$ in $\mathrm{CHCl}_{3}(2-5 \mathrm{~mL}), 2 \mathrm{M}$ sodium acetate $(10 \mathrm{~mL})$ was added and stirred vigorously then a solution of phthaloylglycyl chloride $(1.5 \mathrm{eq})$ in $\mathrm{CHCl}_{3}(3-5 \mathrm{~mL})$, was added to a reaction mixture and left for stirring for $2 \mathrm{~h}$, the organic layer was separated and washed with $\mathrm{Na}_{2} \mathrm{CO}_{3}$ solution three times, the resulting organic layer $\left(\mathrm{CHCl}_{3}\right)$ was dried with $\mathrm{Na}_{2} \mathrm{SO}_{4}$ and concentrated, washed the solid residue with methanol to get the pure product (Scheme 1) [26].

2-(1,3-Dioxoisoindolin-2-yl)- $N, N$-dimethylacetamide

(4a) [27]: Color: White. Yield: $30 \%$. M.p.: $168-170{ }^{\circ} \mathrm{C} . \mathrm{R}_{f}: 0.70\left(\mathrm{CHCl}_{3}\right.$ : EtOAc) (7:3). FT-IR (KBr, v, $\left.\mathrm{cm}^{-1}\right): 1773,1721(\mathrm{C}=0)$ (imide), $1656(\mathrm{C}=0)$ (amide), 1614 (aromatic), $1429(\mathrm{C}-\mathrm{N}), 721$ (ortho subst, Ar. ring). ${ }^{1} \mathrm{H}$ NMR ( $\left.400 \mathrm{MHz}, \mathrm{CDCl}_{3}, \delta, \mathrm{ppm}\right): 2.92$ (s, $3 \mathrm{H}$, $\left.\mathrm{N}-\mathrm{CH}_{3}\right), 3.06\left(\mathrm{~s}, 3 \mathrm{H}, \mathrm{N}-\mathrm{CH}_{3}\right), 4.44\left(\mathrm{~s}, 2 \mathrm{H}, \mathrm{N}-\mathrm{CH}_{2}-\mathrm{C}=0\right), 7.66-7.83$ (m, 4H, Ar-H). ${ }^{13} \mathrm{C}$ NMR $\left(100 \mathrm{MHz}, \mathrm{CDCl}_{3}, \delta, \mathrm{ppm}\right): 35.78(1 \mathrm{C}, \mathrm{N}-$ $\left.\mathrm{CH}_{3}\right), 36.17\left(1 \mathrm{C}, \mathrm{N}-\mathrm{CH}_{3}\right), 39.19\left(1 \mathrm{C}, \mathrm{N}-\mathrm{CH}_{2}-\mathrm{C}=\mathrm{O}\right), 123.4$ (2C, ArC), 132.29 (2C-Ar-C), 133.95 (2C, Ar-C), 165.27 (2C, C=0), 168.0 (1C, C=0). MS (EI, $m / z(\%)): 232\left(\mathrm{M}^{+}, 8.41\right), 160$ (12.49), 133 (3.18), 103 (7.28), 72 (100).

2-(1,3-Dioxoisoindolin-2-yl)- $N, N$-diethylacetamide (4b) [28]: Color: White. Yield: $65 \%$. M.p.: $158-160{ }^{\circ} \mathrm{C} . \mathrm{R}_{f}: 0.80\left(\mathrm{CHCl}_{3}\right.$ :EtOAc) (8:2). FT-IR (KBr, v, cm$\left.{ }^{-1}\right): 1769,1720(\mathrm{C}=0)$ (imide), 1659 (C=0) (amide), 1617 (aromatic), 1464 (C-N), 714 (ortho subst, Ar. ring). ${ }^{1} \mathrm{H}$ NMR (400 MHz, $\left.\mathrm{CDCl}_{3}, \delta, \mathrm{ppm}\right): 1.08-1.129$ (t, $\left.J=5.4 \mathrm{~Hz}, 3 \mathrm{H}, \mathrm{CH}_{2}-\mathrm{CH}_{3}\right), 1.28-1.31\left(\mathrm{t}, J=5.4 \mathrm{~Hz}, 3 \mathrm{H}, \mathrm{CH}_{2}-\mathrm{CH}_{3}\right)$, $3.34-3.36\left(\mathrm{q}, J=3.3 \mathrm{~Hz}, 4 \mathrm{H}, 2\left(\mathrm{CH}_{2}-\mathrm{CH}_{3}\right)\right), 4.45\left(\mathrm{~s}, 2 \mathrm{H}, \mathrm{N}-\mathrm{CH}_{2}-\right.$ $\mathrm{C}=0)$, 7.67-7.85 (m, 4H, Ar-H). ${ }^{13} \mathrm{C}$ NMR $\left(100 \mathrm{MHz}, \mathrm{CDCl}_{3}, \delta\right.$, ppm): $12.87\left(1 \mathrm{C}, \mathrm{CH}_{3}\right), 14.14\left(1 \mathrm{C}, \mathrm{CH}_{3}\right), 39.01\left(1 \mathrm{C}, \mathrm{N}-\mathrm{CH}_{2}-\mathrm{CO}\right)$, 40.77 (1C, N-CH2), 41.30 (1C, N-CH2), 123.39 (2C, $\mathrm{Ar}-\mathrm{C}), 132.32$ (2C, Ar-C), 133.89 (2C, Ar-C), 164.48 (2C, C=0), 168.05 (1C, $\mathrm{C}=0)$. MS (EI, $m / z(\%)): 260\left(\mathrm{M}^{+}, 3.17\right), 161$ (2.04), 133 (2.52), 100 (52.31), 72(100), 58 (5.74).

N,N-Di-sec-butyl-2-(1,3-dioxoisoindolin-2-yl)acetamide (4c): Color: White. Yield: $34 \%$. M.p.: $130-132{ }^{\circ} \mathrm{C}$. Rf: $0.68\left(\mathrm{CHCl}_{3}\right.$ : EtOAc) (8:2). FT-IR (KBr, v, cm-1): 1771, $1719(\mathrm{C}=0)$ (imide), $1647(\mathrm{C}=0)$ (amide), 1629 (aromatic), $1418(\mathrm{C}-\mathrm{N}), 713$ (ortho subst, Ar. ring). ${ }^{1} \mathrm{H}$ NMR $\left(400 \mathrm{MHz}, \mathrm{CDCl}_{3}, \delta, \mathrm{ppm}\right): 0.86-0.88$ (d, $\left.J=6.6 \mathrm{~Hz}, 6 \mathrm{H}, 2 \mathrm{CH}_{3}\right), 1.01-1.03\left(\mathrm{~d}, J=6.6 \mathrm{~Hz}, 6 \mathrm{H}, 2 \mathrm{CH}_{3}\right), 1.96-$ $2.13(\mathrm{~m}, 2 \mathrm{H}, 2 \mathrm{CH}), 3.1-3.2\left(\mathrm{q}, J=7.5 \mathrm{~Hz}, 4 \mathrm{H}, 2 \mathrm{CH}_{2}\right), 4.56(\mathrm{~s}, 2 \mathrm{H}$, $\left.\mathrm{N}-\mathrm{CH}_{2}-\mathrm{CO}\right), 7.79-7.88(\mathrm{~m}, 4 \mathrm{H}, \mathrm{Ar}-\mathrm{H}) .{ }^{13} \mathrm{C}$ NMR $\left(100 \mathrm{MHz}, \mathrm{CDCl}_{3}\right.$, $\delta, \mathrm{ppm}): 20.30\left(2 \mathrm{C}, 2 \mathrm{CH}_{3}\right), 27.64\left(2 \mathrm{C}, 2 \mathrm{CH}_{3}\right), 28.79\left(2 \mathrm{C}, 2 \mathrm{CH}_{2}\right)$, $40.37\left(1 \mathrm{C}, \mathrm{N}-\mathrm{CH}_{2}-\mathrm{CO}\right), 54.64(1 \mathrm{C}, \mathrm{CH}), 55.71(1 \mathrm{C}, \mathrm{CH}), 124.28$ (2C, Ar-C), 133.60 (2C, Ar-C), 135.47 (2C, Ar-C), 168.63 (2C, $\mathrm{C}=0), 169.39$ (1C, $\mathrm{C}=0)$. MS (EI, $m / z(\%)): 316\left(\mathrm{M}^{+}, 11.1\right), 188$ (34.4), 160 (30.8), 133 (13.4), 104 (18.9), 78 (2.9).

2-(1,3-Dioxoisoindolin-2-yl)-N-propylacetamide (4d) [29]: Color: White. Yield: $57 \%$. M.p.: $164-166{ }^{\circ} \mathrm{C}$. Rf: $0.65\left(\mathrm{CHCl}_{3}\right.$ : EtOAc) (8:2). FT-IR (KBr, v, cm-1): $3412(\mathrm{NH})$ (amide), 1774, 1726 (C=0) (imide), 1655 ( $\mathrm{C}=0$ ) (amide), 1629 (aromatic), 1419 (C-N), 712 (ortho subst, Ar. ring). ${ }^{1} \mathrm{H}$ NMR $(400 \mathrm{MHz}$, $\left.\mathrm{CDCl}_{3}, \delta, \mathrm{ppm}\right): 0.89-0.99\left(\mathrm{t}, J=8.1 \mathrm{~Hz}, 3 \mathrm{H}, \mathrm{CH}_{2}-\mathrm{CH}_{3}\right), 1.66(\mathrm{~m}$, $\left.2 \mathrm{H}, \mathrm{CH}_{2}-\mathrm{CH}_{2}-\mathrm{CH}_{3}\right), 3.13-3.33\left(\mathrm{t}, J=6.9 \mathrm{~Hz}, 2 \mathrm{H}, \mathrm{CH}_{2}-\mathrm{CH}_{2}-\mathrm{CH}_{3}\right)$, 4.83 (s, 2H, N-CH $-\mathrm{CO}), 7.49-7.88(\mathrm{~m}, 4 \mathrm{H}, \mathrm{Ar}-\mathrm{H}), 8.02(\mathrm{~s}, 1 \mathrm{H}$, $\mathrm{NH}-$ ). ${ }^{13} \mathrm{C}$ NMR (100 MHz, $\left.\mathrm{CDCl}_{3}, \delta, \mathrm{ppm}\right): 11.87\left(1 \mathrm{C}, \mathrm{CH}_{3}\right), 23.49$
(1C, $\left.\mathrm{CH}_{2}\right), 44.24$ (1C, N-CH $\left.2-\mathrm{CO}\right), 124.31$ (2C-Ar-C), 131.65 (2CAr-C), 135.87 (2C, Ar-C), 169.33 (2C, C=0), 171.79 (1C, C=0). MS (EI, $m / z(\%)): 246\left(\mathrm{M}^{+}, 6.7\right), 205$ (3.4), 188 (5.9), 161 (100), 148 (3.4), 134 (2.2), 105 (22.6).

2-(1,3-Dioxoisoindolin-2-yl)-N-pentylacetamide (4e): Color: White. Yield: $35 \%$. M.p.: $180-182{ }^{\circ} \mathrm{C}$. Rf: $0.78\left(\mathrm{CHCl}_{3}: \mathrm{EtOAc}\right)$ (9:1). FT-IR (KBr, v, cm-1): $3290(\mathrm{NH})$ (amide), 1777, 1728 $(\mathrm{C}=0)$ (imide), 1659 ( $\mathrm{C}=0$ ) (amide), 1617 (aromatic), 1420 (C$\mathrm{N}), 714$ (ortho subst, Ar. ring). ${ }^{1} \mathrm{H}$ NMR $\left(400 \mathrm{MHz}, \mathrm{CDCl}_{3}, \delta\right.$, ppm): 0.74-0.78 (t, $\left.J=6.6 \mathrm{~Hz}, 3 \mathrm{H}, \mathrm{CH}_{3}\right), 1.17\left(\mathrm{~m}, 4 \mathrm{H}, 2 \mathrm{CH}_{2}\right)$, $1.35-1.40\left(\mathrm{t}, J=6.0 \mathrm{~Hz}, 2 \mathrm{H}, \mathrm{CH}_{2}\right), 3.0-3.11\left(\mathrm{t}, J=7.2 \mathrm{~Hz}, 2 \mathrm{H}, \mathrm{CH}_{2}\right)$, $4.19\left(\mathrm{~s}, 2 \mathrm{H}, \mathrm{N}-\mathrm{CH}_{2}-\mathrm{C}=\mathrm{O}\right), 7.61-7.76(\mathrm{~m}, 4 \mathrm{H}, \mathrm{Ar}-\mathrm{H}), 8.02(\mathrm{~s}, 1 \mathrm{H}$, $\mathrm{NH}-$ ). ${ }^{13} \mathrm{C}$ NMR (100 MHz, $\left.\mathrm{CDCl}_{3}, \delta, \mathrm{ppm}\right): 13.65\left(1 \mathrm{C}, \mathrm{CH}_{3}\right), 22.05$ (1C, $\left.\mathrm{CH}_{2}\right), 28.73\left(2 \mathrm{C}, 2 \mathrm{CH}_{2}\right), 39.51\left(1 \mathrm{C}, \mathrm{CH}_{2}\right), 40.19\left(1 \mathrm{C}, \mathrm{N}-\mathrm{CH}_{2}\right.$ CO), 123.27 (2C, Ar-C), 131.78 (2C, Ar-C), 134.06 (2C,Ar-C), $166.31(2 \mathrm{C}, \mathrm{C}=0), 167.87(1 \mathrm{C}, \mathrm{C}=0)$. MS (EI, $m / z(\%)): 274\left(\mathrm{M}^{+}\right.$, 21.3), 205 (18.6), 188 (34.9), 161 (100), 146 (3.8), 133 (56.9), 86 (8.3), 78(8.6).

2-(1,3-Dioxoisoindolin-2-yl)-N-(4-methoxyphenyl)acetamide (4f) [30]: Color: Light grey. Yield: $18 \%$. M.p.: $128-132{ }^{\circ} \mathrm{C}$. Rf: $0.76\left(\mathrm{CHCl}_{3}: \mathrm{EtOAc}\right)$ (9:1). FT-IR $\left(\mathrm{KBr}, v, \mathrm{~cm}^{-1}\right): 3416(\mathrm{NH})$ (amide), 1775, 1729 ( $\mathrm{C}=0$ ) (imide), 1660 ( $\mathrm{C}=0$ ) (amide), 1643 (aromatic), 1414 (C-O), 716 (ortho subst, Ar. ring). ${ }^{1} \mathrm{H}$ NMR $\left(400 \mathrm{MHz}, \mathrm{CDCl}_{3}, \delta, \mathrm{ppm}\right): 3.66\left(\mathrm{~s}, 3 \mathrm{H}, \mathrm{O}-\mathrm{CH}_{3}\right), 4.37(\mathrm{~s}, 2 \mathrm{H}, \mathrm{N}-$ $\mathrm{CH}_{2}-\mathrm{CO}$ ), 6.71-6.73 (d, $\left.J=6.6 \mathrm{~Hz}, 2 \mathrm{H}, \mathrm{Ar}-\mathrm{H}\right), 7.25-7.34(\mathrm{~m}, 4 \mathrm{H}$, $\mathrm{Ar}-\mathrm{H}$ ), 7.76-7.78 (d, $J=2.1 \mathrm{~Hz}, 2 \mathrm{H}, \mathrm{Ar}-\mathrm{H}), 7.25$ (s, $1 \mathrm{H},-\mathrm{NH}-{ }^{13}{ }^{13} \mathrm{C}$ NMR $\left(100 \mathrm{MHz}, \mathrm{CDCl}_{3}, \delta, \mathrm{ppm}\right): 41.71\left(1 \mathrm{C}, \mathrm{N}-\mathrm{CH}_{2}-\mathrm{CO}\right), 55.47$ (1C, $\left.\mathrm{OCH}_{3}\right), 114.21$ (2C, Ar-C), 121.86 (2C, Ar-C), 123.74 (2C, Ar-C), 134.35 (2C, Ar-C), 135.40 (2C, Ar-C), 139.40 (1C, Ar-C $\mathrm{NH}), 156.50\left(1 \mathrm{C}, \mathrm{Ar}-\mathrm{COCH}_{3}\right), 165.55$ (2C, C=0), 167.82 (1C $\mathrm{C}=0)$. MS (EI, $m / z(\%)): 310\left(\mathrm{M}^{+}, 99.1\right), 161(50.9), 160$ (100), 133 (13.0), 123 (86.3), 108 (17.8), 78 (14.8).

2-(1,3-Dioxoisoindolin-2-yl)-N-(pyridin-2-yl)acetamide $\quad \mathbf{4} \mathbf{g})$ [28]: Color: Light yellow. Yield: $21 \%$. M.p.: $134-138{ }^{\circ} \mathrm{C}$. Rf: 0.76 ( $\mathrm{CHCl}_{3}: \mathrm{EtOAc}$ ) (9:1). FT-IR (KBr, v, cm$\left.{ }^{-1}\right): 3414(\mathrm{NH})$ (amide), 1771-1722 ( $\mathrm{C}=0$ ) (imide), $1693(\mathrm{C}=0)$ (amide), 1662 (aromatic), 1421 (C-N), 714 (ortho subst, Ar. ring). ${ }^{1} \mathrm{H}$ NMR (400 $\left.\mathrm{MHz}, \mathrm{CDCl}_{3}, \delta, \mathrm{ppm}\right): 4.50\left(\mathrm{~s}, 2 \mathrm{H}, \mathrm{N}-\mathrm{CH}_{2}-\mathrm{CO}\right), 7.67-7.84(\mathrm{~m}, 4 \mathrm{H}$, Ar-H), 7.76-7.77 (d, $J=5.6 \mathrm{~Hz}, 1 \mathrm{H}, \mathrm{Ar}-\mathrm{H}), 7.50(\mathrm{~d}, J=1.6 \mathrm{~Hz}, 1 \mathrm{H}$, Ar-H), 8.05-8.07 (d, $J=6.4 \mathrm{~Hz}, 1 \mathrm{H}, \mathrm{Ar}-\mathrm{H}), 8.16-8.17$ (d, $J=4.0$ $\mathrm{Hz}, 1 \mathrm{H}, \mathrm{Ar}-\mathrm{H}), 9.30$ (s, 1H, -NH-). ${ }^{13} \mathrm{C}$ NMR $\left(100 \mathrm{MHz}, \mathrm{CDCl}_{3}, \delta\right.$, ppm): 41.39 (1C, N-CH $-\mathrm{CO}), 110.04$ (1C, Ar-C), 114.49 (1C, ArC), 123.69 (2C, Ar-C), 132.01 (2C, Ar-C), 134.26 (2C, Ar-C), 138.55 (1C, Ar-C), 147.65 (1C, Ar-C), 150.99 (1C, Ar-C), 164.76 (1C, $\mathrm{C}=0), 167.72$ (2C, $\mathrm{C}=0)$. MS (EI, $m / z(\%)): 281\left(\mathrm{M}^{+}, 23.8\right)$, 188 (4.1), 160 (99.5), 134 (56.5), 121 (100), 105 (10.4), 78 (20.7).

2-(1,3-Dioxoisoindolin-2-yl)- $N$-(2-hydroxyethyl)acetamide (4h) [31]: Color: White. Yield: $87 \%$. M.p.: $110-112{ }^{\circ} \mathrm{C}$. Rf: 0.65 ( $\left.\mathrm{CHCl}_{3}: \mathrm{MeOH}\right)(8: 2)$. FT-IR $\left(\mathrm{KBr}, v, \mathrm{~cm}^{-1}\right): 3472(\mathrm{NH})$ (amide), $3590(\mathrm{OH})$ (alcohol), $1770(\mathrm{C}=0)$ (imide), $1699(\mathrm{C}=0)$ (amide), 1650 (aromatic), 1056 (C-0), 725 (ortho subst, Ar. ring). ${ }^{1} \mathrm{H}$ NMR (400 MHz, $\left.\mathrm{CDCl}_{3}, \delta, \mathrm{ppm}\right): 3.73\left(\mathrm{t}, J=5.4 \mathrm{~Hz}, 2 \mathrm{H}, \mathrm{CH}_{2}\right), 3.79$ $\left(\mathrm{t}, J=5.1 \mathrm{~Hz}, 2 \mathrm{H}, \mathrm{CH}_{2}\right), 4.50(\mathrm{~s}, 1 \mathrm{H}, \mathrm{OH}), 4.84\left(\mathrm{~s}, 2 \mathrm{H}, \mathrm{N}-\mathrm{CH}_{2}-\mathrm{CO}\right)$, 7.75-7.84 (m, 4H, Ar-H), 8.02 (s, $1 \mathrm{H},-\mathrm{NH}-) .{ }^{13} \mathrm{C}$ NMR (100 MHz, $\left.\mathrm{CDCl}_{3}, \delta, \mathrm{ppm}\right): 29.14\left(1 \mathrm{C}, \mathrm{CH}_{2}\right), 39.15\left(1 \mathrm{C}, \mathrm{CH}_{2}\right), 44.23(1 \mathrm{C}, \mathrm{N}-$ $\mathrm{CH}_{2}$-CO), 124.10 (2C, Ar-C), 136.09 (2C, Ar-C), 140.96 (2C, ArC), $165.35(2 \mathrm{C}, \mathrm{C}=0), 169.05(1 \mathrm{C}, \mathrm{C}=0)$. FAB MS $(+, m / z): 249$ $[\mathrm{M}+\mathrm{H}]$. 


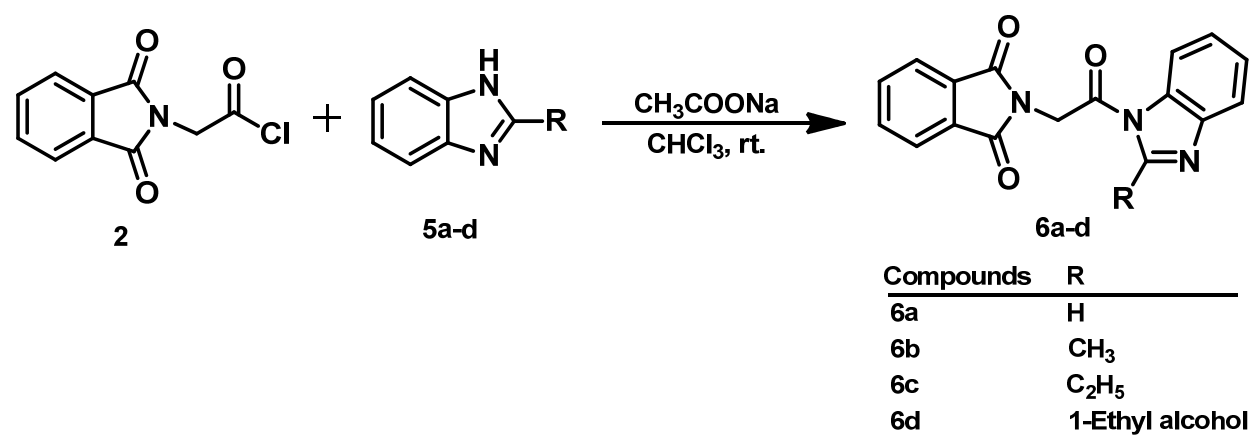

Scheme 3

\subsubsection{General procedure for the preparation of benzimidazole derivatives of $N$-phthaloylglycine (6a-d)}

To a solution of Benzimidazole derivatives (5a-d) in $\mathrm{CHCl}_{3}$ (2-5 mL), $2 \mathrm{M}$ sodium acetate $(10 \mathrm{~mL})$ was added and stirred vigorously then a solution of phthaloylglycyl chloride (1.5 eq) in $\mathrm{CHCl}_{3}(3-5 \mathrm{~mL})$, was added to a reaction mixture and left for stirring for $2 \mathrm{~h}$, the organic layer was separated and washed with aqueous $\mathrm{Na}_{2} \mathrm{CO}_{3}$ solution three times, the resulting organic layer was dried with $\mathrm{Na}_{2} \mathrm{SO}_{4}$ and concentrated, washed the solid residue with methanol to get the pure product (Scheme 3) [26].

2-[2-(1H-Benzimidazol-1-yl)-2-oxoethyl]-1H-isoindole-1,3 (2H)-dione (6a): Color: White. Yield: $32 \%$. M.p.: $230-232{ }^{\circ} \mathrm{C}$. Rf: 0.53 ( $\left.\mathrm{CHCl}_{3}: \mathrm{MeOH}\right)$ (9:1). FT-IR (KBr, v, $\left.\mathrm{cm}^{-1}\right): 1776,1715$ $(\mathrm{C}=0)$ (imide), $1674(\mathrm{C}=0)$ (amide), 1650 (aromatic), 1423 (C$\mathrm{N}), 725$ (ortho subst, Ar. ring). ${ }^{1} \mathrm{H}$ NMR $\left(400 \mathrm{MHz}, \mathrm{CDCl}_{3}, \delta\right.$, ppm): $5.13\left(\mathrm{~s}, 2 \mathrm{H}, \mathrm{N}-\mathrm{CH}_{2}-\mathrm{C}=0\right)$, 7.75-7.80 (m, 4H, Ar-H), 7.82$7.91(\mathrm{~m}, 4 \mathrm{H}, \mathrm{Ar}-\mathrm{H}), 8.52(\mathrm{~s}, 1 \mathrm{H}, \mathrm{N}-\mathrm{CH}=\mathrm{N}) .{ }^{13} \mathrm{C}$ NMR $(100 \mathrm{MHz}$, $\left.\mathrm{CDCl}_{3}, \delta, \mathrm{ppm}\right): 40.52$ (1C, N-CH2-O), 115.27 (1C, Ar-C), 120.06 (1C, Ar-C), 123.66 (2C, Ar-C), 125.51 (1C, Ar-C), 126.26 (1C, ArC), 130.90 (1C, Ar-C), 131.58 (2C, Ar-C), 134.13 (2C, Ar-C), $140.33(1 \mathrm{C}, \mathrm{N}-\mathrm{CH}=\mathrm{N}), 142.76$ (1C, Ar-C), 163.85 (1C, C=0), 167.40 (1C, Ar-C). MS (EI, $m / z(\%)): 305\left(\mathrm{M}^{+}, 7.5\right), 188$ (12.4), 160 (100), 119 (29.42), 104 (9.96), 78 (4.04).

2-[2-(2-Methyl-1H-benzimidazol-1-yl)-2-oxoethyl]-1H-iso indole-1,3(2H)-dione (6b): Color: White. Yield: 32\%. M.p: 232$234{ }^{\circ} \mathrm{C} . \mathrm{R}_{f}: 0.65\left(\mathrm{CHCl}_{3}: \mathrm{EtOH}\right)$ (8:2). FT-IR (KBr, $\left.v, \mathrm{~cm}^{-1}\right):$ 1769, $1713(\mathrm{C}=0)$ (imide), $1686(\mathrm{C}=0)$ (amide), 1614 (aromatic), 1425 (C-N), 725 (ortho subst, Ar. ring). ${ }^{1} \mathrm{H}$ NMR $(400 \mathrm{MHz}$, $\left.\mathrm{CDCl}_{3}, \delta, \mathrm{ppm}\right): 2.81\left(\mathrm{~s}, 3 \mathrm{H}, \mathrm{CH}_{3}\right), 5.10\left(\mathrm{~s}, 2 \mathrm{H}, \mathrm{N}-\mathrm{CH}_{2}-\mathrm{CO}\right), 7.13-$ 7.37 (m, 4H-Ar-H), 7.65-7.78 (m, 4H, Ar-H). ${ }^{13} \mathrm{C}$ NMR (100 MHz, $\left.\mathrm{CDCl}_{3}, \delta, \mathrm{ppm}\right): 18.91\left(1 \mathrm{C}, \mathrm{CH}_{3}\right), 42.84\left(1 \mathrm{C}, \mathrm{N}-\mathrm{CH}_{2}-\mathrm{CO}\right), 113.76$ (1C, Ar-C), 119.88 (1C, Ar-C), 123.50 (2C, Ar-C), 124.93 (1C, ArC), 125.06 (1C, $\mathrm{Ar}-\mathrm{C}), 131.78$ (1C, $\mathrm{Ar}-\mathrm{C}), 134.21$ (2C, $\mathrm{Ar}-\mathrm{C}$ ), 142.23 (1C, N-CH=N), 153.22 (2C, Ar-C), 165.63 (1C, C=0), 167.40 (2C, C=0). MS (EI, $m / z(\%)): 319\left(\mathrm{M}^{+}, 4.6\right), 291(11.5)$, 160 (100), 147 (34.2), 132 (55.8), 104 (45.5), 78 (3.5).

2-[2-(2-Ethyl-1H-benzimidazol-1-yl)-2-oxoethyl]-1H-iso indole-1,3(2H)-dione (6c): Color: White. Yield: 30\%. M.p.: 208$210^{\circ} \mathrm{C} . \mathrm{R}_{f}: 0.65\left(\mathrm{CHCl}_{3}: \mathrm{EtOAc}\right)(8: 2)$. FT-IR $\left(\mathrm{KBr}, \mathrm{v}, \mathrm{cm}^{-1}\right): 1774$, $1710(\mathrm{C}=0)$ (imide), $1686(\mathrm{C}=0)$ (amide), 1650 (aromatic), 1425 (C-N), 1056 (C-O), 725 (ortho subst, Ar. ring). ${ }^{1} \mathrm{H}$ NMR (400 MHz, $\left.\mathrm{CDCl}_{3}, \delta, \mathrm{ppm}\right): 1.09-1.28\left(\mathrm{t}, J=7.2 \mathrm{~Hz}, 3 \mathrm{H}, \mathrm{CH}_{3}\right)$, $3.02-3.08$ (q, $J=7.2 \mathrm{~Hz}, 2 \mathrm{H}, \mathrm{CH}_{2}-\mathrm{CH}_{3}$ ), 5.03 (s, $2 \mathrm{H}, \mathrm{N}-\mathrm{CH}_{2}-\mathrm{CO}$ ), 7.24-7.27 (m, 4H, Ar-H), 7.76-7.80 (m, 4H, Ar-H). ${ }^{13} \mathrm{C}$ NMR (100 $\left.\mathrm{MHz}, \mathrm{CDCl}_{3}, \delta, \mathrm{ppm}\right): 11.83\left(1 \mathrm{C}, \mathrm{CH}_{3}\right), 25.28\left(1 \mathrm{C}, \mathrm{CH}_{2}\right), 42.97$ (1C, N-CH $-\mathrm{CO}$ ), 113.47 (1C, Ar-C), 120.01 (1C, Ar-C), 123.26 (1C, Ar-C), 123.48 (1C, Ar-C), 124.88 (2C, Ar-C), 131.57 (1C, ArC), 134.0 (1C, Ar-C), 134.48 (2C, Ar-C), $142.23(\mathrm{~N}-\mathrm{CH}=\mathrm{N})$, 158.44 (1C, C=0), 167.47 (2C, C=0). MS (EI, $m / z(\%)$ ): 333.1 $\left(\mathrm{M}^{+}, 58\right), 305$ (4.3), 188 (5.4), 173 (46.6), 160 (100), 147 (96), 133 (11.4), 78 (2).
2-(2-(2-(1-Hydroxyethyl)-1H-benzo[d]imidazol-1-yl)-2-oxo ethyl)isoindoline-1,3-dione (6d): Color: White. Yield: 32\%. M.p.: 180-200 ${ }^{\circ} \mathrm{C} . \mathrm{R}_{f}: 0.65\left(\mathrm{CHCl}_{3}: \mathrm{EtOAc}\right)(8: 2)$. FT-IR $\left(\mathrm{KBr}, v, \mathrm{~cm}^{-1}\right)$ : 3590-2625 (OH) (alcohol), 1775, 1722 (C=0) (imide), 1699 $(\mathrm{C}=0)$ (amide), 1650 (aromatic), $1420(\mathrm{C}-\mathrm{N}), 1056$ (C-O), 725 (ortho subst, Ar. ring). ${ }^{1} \mathrm{H}$ NMR $\left(400 \mathrm{MHz}, \mathrm{CDCl}_{3}, \delta\right.$, ppm): 1.76 $\left(\mathrm{d}, J=6.6 \mathrm{~Hz}, 3 \mathrm{H}, \mathrm{CH}_{3}\right), 3.76(\mathrm{~s}, 1 \mathrm{H}, \mathrm{OH}), 4.64\left(\mathrm{~s}, 2 \mathrm{H}, \mathrm{N}-\mathrm{CH}_{2}-\mathrm{CO}\right)$, $5.25\left(\mathrm{q}, J=16.8 \mathrm{~Hz}, 1 \mathrm{H}, \mathrm{CH}-\mathrm{CH}_{3}\right), 7.66-7.72(\mathrm{~m}, 4 \mathrm{H}, \mathrm{Ar}-\mathrm{H}), 7.90-$ $7.94(\mathrm{~m}, 4 \mathrm{H}, \mathrm{Ar}-\mathrm{H}) .{ }^{13} \mathrm{C}$ NMR $\left(100 \mathrm{MHz}, \mathrm{CDCl}_{3}, \delta, \mathrm{ppm}\right): 18.41$ (1C, $\left.\mathrm{CH}_{3}\right), 42.95$ (1C, $\left.\mathrm{CH}-\mathrm{OH}\right), 68.77$ (1C, N-CH $\left.-\mathrm{CO}\right), 113.24$ (1C, Ar-C), 121.80 (1C, Ar-C), 123.50 (1C, Ar-C), 123.98 (1C, Ar-C), 125.28 (2C, Ar-C), 131.47 (2C, Ar-C), 131.97 (1C, Ar-C), 134.0 (2C, Ar-C), 134.5 (1C, Ar-C), 142.58 (1C, N-C=N), 154.51 (1C, $\mathrm{C}=0), 167.47$ (2C, $\mathrm{C}=0)$. MS (EI, $m / z(\%)): 349\left(\mathrm{M}^{+}, 23\right), 331$ (8.1), 305 (26.4), 288 (36.9), 273 (67.5), 258 (17.5), 187 (15.6), 160 (100), 145 (77.9), 132 (27.1), 104 (42.3), 78(2).

\subsubsection{Antimicrobial activity assay}

Disc diffusion method was adopted to determine the antimicrobial activity of compounds $4 \mathbf{a}-\mathbf{h}$ and $\mathbf{6 a}-\mathbf{d}$ using $6 \mathrm{~mm}$ sterile filter disc. Clinical isolates of Staphylococcus aureus (KIBGE: MBSA-01 to MBSA-43), strains of Staphylococcus aureus (ATCC 6538), Enterococcus faecalis (ATCC 29212), Bacillus cereus (ATCC 11778), Salmonella typhi (ATCC 3632) and Pseudomonas aeruginosa (KIBGE: IB-67) were used [32].

Solutions of the compounds were prepared in chloroform (Merck, Germany), concentrations ranging from 7.5 to $350 \mu \mathrm{g}$ per disc. As positive control antimicrobial susceptibility discs of vancomycin $(30 \mu \mathrm{g})$, oxcillin $(1 \mu \mathrm{g})$ and ciprofloxacin $(5 \mu \mathrm{g})$ (Oxoid, UK) were used, whereas chloroform disc were used as negative control.

Microorganisms were culture in Luria Broth (Oxoid, UK) at $35^{\circ} \mathrm{C}$, overnight. $1.5 \times 10^{6}$ cells $/ \mathrm{mL}(0.5 \mathrm{McF}$ arland index $)$ were inoculated on Mueller Hinton Agar (Oxoid, UK) $[33,34]$. On each plate filter disc with given compounds, positive and negative control discs were placed. After an incubation period of $24 \mathrm{~h}$ at $35-37{ }^{\circ} \mathrm{C}$, zone of inhibition (in $\mathrm{mm}$ ) were measured under bright light and on non-reflecting background.

\section{Result and discussions}

In the present study, two series of $N$-phthaloylglycine derivatives (4a-h and 6a-d) were prepared under SchottenBaumann reaction conditions and were evaluated for antibacterial activity against methicillin-resistant $S$. aureus (MRSA) and vancomycin-resistant $S$. aureus (VRSA). In disc diffusion method, antimicrobial activities were evaluated by measuring the diameter of zone of inhibition against test organisms and minimal inhibitory concentration (MIC), the values of which are displayed in Tables 1,2 and 3 , respectively [29]. Almost all the compounds showed some activity against VRSA and MRSA. 
Table 1. Antibacterial activity of $N$-phthaloylglycine derivatives (i.e. 4a-h, and 6a-d) against vancomycin resistant Staph. aureus (VRSA) (N = 40).

\begin{tabular}{|c|c|c|c|c|c|}
\hline \multirow[t]{2}{*}{ Compounds } & \multicolumn{2}{|c|}{ Zone of inhibition (mm) } & \multirow{2}{*}{$\begin{array}{l}\text { No inhibition } \\
\text { (Number of strains) }\end{array}$} & \multirow{2}{*}{$\begin{array}{l}\text { Inhibition } \\
\text { (Number of strains) }\end{array}$} & \multirow[t]{2}{*}{ \% Inhibition } \\
\hline & Minimum & Maximum & & & \\
\hline$\overline{4 a}$ & $2 \pm 0.07$ & $10 \pm 1.5$ & 29 & 11 & 27.50 \\
\hline $4 b$ & $3 \pm 0.10$ & $8 \pm 1.3$ & 10 & 30 & 75.00 \\
\hline $4 c$ & $2 \pm 0.08$ & $10 \pm 1.8$ & 08 & 32 & 80.00 \\
\hline $4 d$ & $3 \pm 0.09$ & $6 \pm 1.1$ & 13 & 27 & 67.50 \\
\hline $4 \mathrm{e}$ & $3 \pm 0.06$ & $8 \pm 1.8$ & 11 & 29 & 72.50 \\
\hline $4 \mathrm{f}$ & $2 \pm 0.04$ & $6 \pm 1.4$ & 12 & 28 & 70.00 \\
\hline $4 \mathrm{~g}$ & $2 \pm 0.06$ & $8 \pm 1.6$ & 10 & 30 & 75.00 \\
\hline $4 \mathrm{~h}$ & $3 \pm 0.80$ & $8 \pm 1.1$ & 18 & 22 & 55.00 \\
\hline $6 a$ & $2 \pm 0.04$ & $8 \pm 1.8$ & 30 & 10 & $25: 00$ \\
\hline $6 b$ & $3 \pm 0.10$ & $8 \pm 1.5$ & 25 & 15 & 37.50 \\
\hline $6 c$ & $3 \pm 0.08$ & $6 \pm 0.9$ & 15 & 25 & 62.50 \\
\hline $6 \mathrm{~d}$ & $2 \pm 0.06$ & $8 \pm 1.1$ & 23 & 17 & 42.50 \\
\hline
\end{tabular}

Table 2. Antibacterial activity of $N$-phthaloylglycine derivatives (i.e. 4a-h, and 6a-d) against methicillin resistant Staph. aureus (MRSA) (N = 44).

\begin{tabular}{|c|c|c|c|c|c|}
\hline \multirow[t]{2}{*}{ Compounds } & \multicolumn{2}{|c|}{ Zone of inhibition (mm) } & \multirow{2}{*}{$\begin{array}{l}\text { No inhibition } \\
\text { (Number of strains) }\end{array}$} & \multirow{2}{*}{$\begin{array}{l}\text { Inhibition } \\
\text { (Number of strains) }\end{array}$} & \multirow[t]{2}{*}{ \% Inhibition } \\
\hline & Minimum & Maximum & & & \\
\hline $4 a$ & $3 \pm 0.10$ & $6 \pm 0.8$ & 18 & 26 & 59.09 \\
\hline $4 b$ & $1 \pm 0.07$ & $8 \pm 0.9$ & 17 & 27 & 61.36 \\
\hline $4 c$ & $2 \pm 0.08$ & $6 \pm 0.7$ & 12 & 32 & 72.72 \\
\hline $4 d$ & $2 \pm 0.04$ & $6 \pm 0.8$ & 10 & 34 & 77.27 \\
\hline $4 \mathrm{e}$ & $1 \pm 0.06$ & $4 \pm 0.5$ & 18 & 26 & 59.09 \\
\hline $4 f$ & $1 \pm 0.04$ & $7 \pm 0.6$ & 18 & 26 & 59.09 \\
\hline $4 \mathrm{~g}$ & $2 \pm 0.10$ & $9 \pm 0.8$ & 15 & 29 & 65.90 \\
\hline $4 \mathrm{~h}$ & $1 \pm 0.08$ & $8 \pm 0.7$ & 19 & 25 & 56.81 \\
\hline $6 a$ & $1 \pm 0.09$ & $5 \pm 0.2$ & 12 & 32 & 72.72 \\
\hline $6 \mathrm{~b}$ & $2 \pm 0.08$ & $4 \pm 0.09$ & 18 & 26 & 59.09 \\
\hline $6 c$ & $1 \pm 0.09$ & $5 \pm 0.4$ & 16 & 28 & 63.63 \\
\hline $6 d$ & $3 \pm 0.07$ & $5 \pm 0.08$ & 10 & 34 & 77.27 \\
\hline
\end{tabular}

Table 3. Antibacterial activity of $N$-phthaloylglycine derivatives (i.e. 4a-h, and 6a-d) against various strains of Staph. aureus.

\begin{tabular}{lll}
\hline Compounds & MIC $(\mathbf{m g} / \mathbf{L}), \mathbf{V R S A}, \mathbf{( N = 4 0 )}$ & MIC (mg/L), MRSA, (N = 44) \\
\hline 4a & 0.6 & 2.5 \\
4b & 4.0 & 3.6 \\
4c & 5.5 & 7.0 \\
4d & 4.2 & 6.3 \\
4e & 0.7 & 2.9 \\
4f & 7.5 & 4.2 \\
4g & 3.6 & 0.8 \\
4h & 0.5 & 1.6 \\
6a & 1.5 & 8.2 \\
6b & 2.4 & 1.5 \\
6c & 3.0 & 3.2 \\
6d & 2.0 & 4.6 \\
\hline Vancomycin (Standard drug) & 4.0 & - \\
Methicillin (Standard drug) & - & 4.0 \\
\hline
\end{tabular}

Compound $\mathbf{4 c}$, was found to be the most effective against the highest number of clinical isolates of VRSA (80\%), with an inhibitory zone ranging from 2 to $6 \mathrm{~mm}$ (Table 1). Compounds $\mathbf{6 a}$ and $\mathbf{4 c}$ were found to be the most effective against highest number of clinical isolates of MRSA (77.7\%), with a zone of inhibition ranging from 2 to $10 \mathrm{~mm}$ (Table 2).

In particular, compounds $4 \mathrm{~h}$ (MIC, $0.5 \mathrm{mg} / \mathrm{L}$ ), $4 \mathrm{a}$ (MIC, 0.6 $\mathrm{mg} / \mathrm{L}$ ), and $4 \mathrm{e}$ (MIC, $0.7 \mathrm{mg} / \mathrm{L}$ ) were found to be 6 to 8 fold more potent against VRSA as compared to the standard vancomycin (MIC, $4.0 \mathrm{mg} / \mathrm{L}$ ). Compounds $4 \mathrm{~g}$, $6 \mathbf{b}$ and $4 \mathbf{h}$ displayed excellent activity against MRSA (MIC, 0.8, 1.5 and 1.6 $\mathrm{mg} / \mathrm{L}$, respectively) as compared to the standard methicillin (MIC, $4.0 \mathrm{mg} / \mathrm{L}$ ) (Table 3). Moreover, compounds 4c, 4d, 6a, and $\mathbf{6 d}$ showed significant growth inhibitory activity against most of the tested strains for MRSA (Table 2), whereas compounds 4b, 4c and 4g, showed significant growth inhibitory action against most of the tested strains for VRSA (Table 1). Interestingly, acylic amides provide the most potent analogs against VRSA.

The compound $4 \mathrm{~h}$ is found to be the most active, as it exhibited an MIC of $0.5 \mathrm{mg} / \mathrm{L}$ against VRSA, and MIC of 1.6 $\mathrm{mg} / \mathrm{L}$ against MRSA, as well (Table 3). This observation indicates that the presence of hydroxyl group enhances the antimicrobial activity when compared to $\mathbf{4 d}$ that lacks the $\mathrm{OH}$ group. The antibacterial activity of $\mathrm{N}$-phthaloylglycine derivatives (i.e. $\mathbf{4 a - h}$ and 6a-d) were also illustrated graphically in Figures 1, 2 and 3.

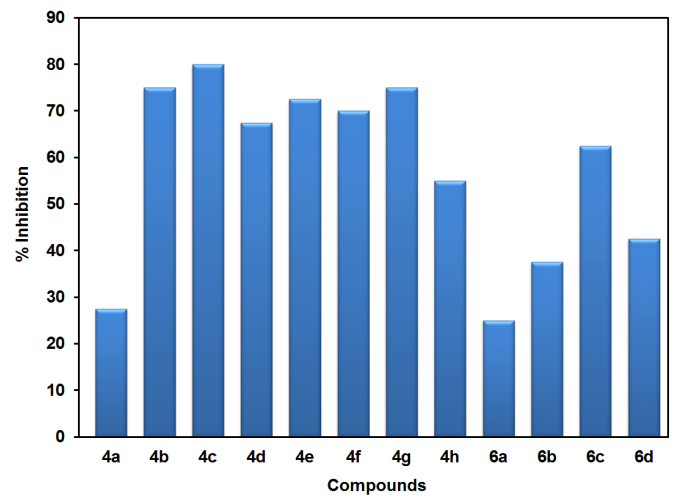

Figure 1. Antibacterial activity of $N$-phthaloylglycine derivatives (i.e. $\mathbf{4 a - h}$, and 6a-d) against vancomycin resistant Staph. aureus (VRSA).

\section{Conclusion}

The development of antimicrobial resistance in many pathogenic microbes possesses one of the most serious 
problems in the control of infectious diseases. In this context, two series of the $\mathrm{N}$-phthaloylglycine derivative were synthesized and evaluated against in vitro antibacterial activity. The compounds $\mathbf{4 h}, \mathbf{4 e}$, and $\mathbf{4 a}$ were found to be 6 to 8 fold more potent against VRSA as compare to the standard vancomycin. The results suggest that the $N$-phthaloylglycine derivatives are interesting lead molecules for further studies. More extensive work is still needed to confirm the preliminary results and mode of action to design and synthesize the potential antimicrobial agents.

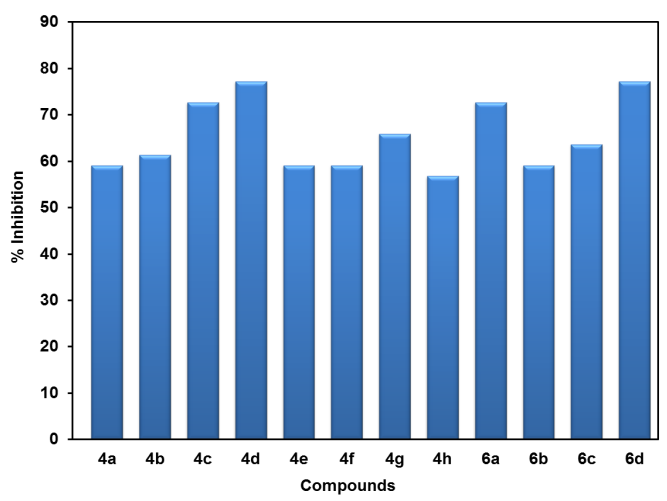

Figure 2. Antibacterial activity of $N$-phthaloylglycine derivatives (i.e. 4a-h, and 6a-d) against methicillin resistant Staph. aureus (MRSA).

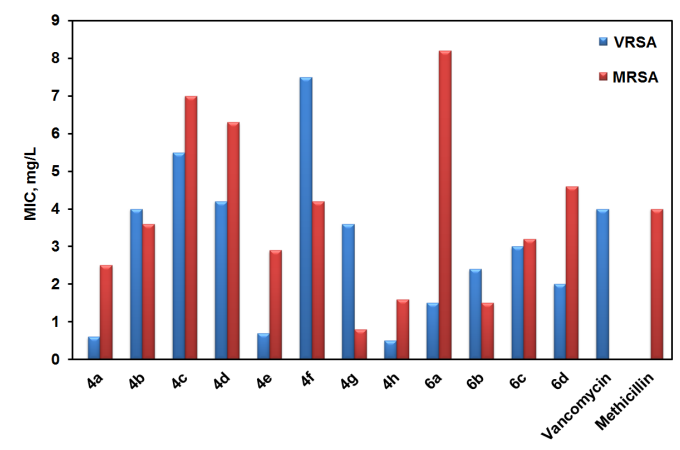

Figure 3. Antibacterial activity of $N$-phthaloylglycine derivatives (i.e. 4a-h, and 6a-d) against various strains of Staph. aureus.

\section{Acknowledgement}

The authors would like to thank the Hussain Ebrahim Jamal Research Institute of Chemistry, International Center for Chemical and Biological Sciences, University of Karachi, for the spectral analysis and the Karachi Institute of Biotechnology and Genetic Engineering (KIBGE), University of Karachi for conducting the antibacterial assay.

\section{References}

[1]. Beceiro, A.; Tomas, M.; Bou, G. Clin. Microbiol. Rev. 2013, 26, 185-230.

[2]. Bishnoi, A.; Singh, S.; Tiwari, A. K.; Sethi, A.; Tripathi, C. M. Med. Chem. 2013, 9, 45-52.

[3]. Batabyal, B.; Kundu , G. K. R.; Biswas, S. I. Res. J. Biological Sci. 2012, 1, 7, 65-71.

[4]. Kobayashi, S. D.; Musser, J. M.; DeLeo, F. R. mBio. 2012, 3, e00170-12.

[5]. Chambers, H. F.; DeLeo, F. R. Nat. Rev. Microbiol. 2009, 7, 629-641

[6]. Grundmann, H.; Aires-de-Sousa, M.; Boyce, J.; Tiemersma, E. Lancet 2006, 368, 874-885.

[7]. Klevens, R. M.; Morrison, M. A.; Nadle, J.; Petit, S.; Gershman, K.; Ray, S.; Harrison, L. H.; Lynfield, R.; Dumyati, G.; Townes, J. M.; Craig, A. S.; Zell, E. R.; Fosheim, G. E.; McDougal, L. K.; Carey, R. B.; Fridkin, S. K. JAMA 2007, 298, 1763-1771.

[8]. Gravatt, G. L.; Baguley, B. C.; Wilson. J. Med. Chem. 1994, 37, 43384345.
[9]. Ahamed, A. J.; Kaliapillai, N. V.; Bathey, R. V.; Govindaraj, V. Orbital Elec. J. Chem. 2009, 1, 306-309.

[10]. Julijay, M. S.; Zduka, C. Acta. Pharm. 2005, 55, 387-399.

[11]. Neto, M. D. C.; Propheric, M. C. F.; Neto, B. B. J. Braz. Chem. Soc. 1993, 4 139-142.

[12]. Antunes, R.; Batista, H.; Srivastava, R. M.; Thomas, G.; Araujo, C. C. Bioorg. Med. Chem. Lett. 1998, 8, 3071-3076.

[13]. Brana, M. F.; Ramose, A. Curr. Med. Chem. 2001, 1, 237-255.

[14]. Cyril, O. U.; Didier, M. L.; Johan, W. G.; Sriba, K. E. Arch. Pharm. Chem. 2001, 334, 323-331.

[15]. Nilotpal, B.; Rupam, J. S.; Andrei, S. B.; Jubaraj, B. B. Polyhedron 2006 $25,17-24$.

[16]. Nilotpal, B.; Rupam, J. S.; Andrei, S. B.; Jubaraj, B. B. J. Mol. Struct. 2006 791, 122-130.

[17]. Shariat, M.; Abdollahi, S. Molecules 2004, 9, 705-712.

[18]. Uzma, Y.; Mohammad, K. T.; Mohammad, H. B.; Naveeda, Y.; Madeleine, H. Acta. Cryt. E 2008, 64, o476-0477.

[19]. Wagner, E. C.; Millett, W. H. Org. Synth. Coll. 1943, 2, 65-67.

[20]. Laszlo, K.; Barbra, C. Strategic application of named reactions in organic synthesis Elsevier Academic Press, 2005.

[21]. Wilcox, C. F. Experimental Organic Chemistry, $5^{\text {th }}$ edition Macmillan Publishing Company, New York, 1984.

[22]. Karami, B.; Ghashghaee, V.; Khodobakhshi, S. Chin. J. Chem. 2012, 30, 959-964.

[23]. Navneet, K.; Pratima, S.; Aastha, P.; Prasand, A. Int. J. Chem. Pharm. Sci. 2013, 14, 12-18.

[24]. Anshul, C.; Gurpreet, K.; Anil, K. S. Int. J. Pharm. Phytopharmacol. Res. 2012, 2, 148-159.

[25]. Kalirajan, R.; Leela, R.; Jubie, S.; Gowramma, B.; Gomathy, S.; Sankar, S.; Elango, K. Ind. J. Pharm. Edu. Res. 2010, 44, 358-362.

[26]. Haishan, W.; Ganesan, A. J. Org. Chem. 2000, 65, 1022-1030.

[27]. Jennifer, D. K.; Philip, M.; Edward, G. M. Tetrahedron Lett. 2001, 42, 627-629.

[28]. Celia, B. S.; Maria, I. A.; Isabel, A. P. J. Heterocyclic Chem. 1985, 22, 577 581.

[29]. Andreichikov, Yu. S.; Zalesov, V. V.; Podushkina, N. A. Khim. Farm. Zh. 1980, 14, 25-30.

[30]. Ashok, P.; Ganguly, S.; Murugesan, S. Der Pharma Chem. 2013, 5, 10 19.

[31]. Smith, E. C.; Althouse, P. M.; Shigley, J. W. J. Am. Oilchem. Soc. 1960, 37, 288-290.

[32]. Bauer, A. W.; Kirby, E.; Sherris, E. M.; Turk, M. Am. J. Clin. Pathol. 1966 45, 493-496

[33]. National Committee for Clinical Laboratory Standards (NCCLS) National committee for clinical laboratory standards for antimicrobial disc susceptibility test: approved standards. $6^{\text {th }}$ edition. NCCLS, Wayen, Pennsylvania, USA, 2005.

[34]. Chandrasekaran, M.; Venkatesalu, V. J. Ethonopharmacol. 2005, 91 105-108. 